\title{
Every urologist and oncologist should know about treating sexual and gender minority prostate cancer patients: translating research findings into clinical practice
}

\author{
B. R. Simon Rosser ${ }^{1}$, G. Nic Rider ${ }^{2}$, Aditya Kapoor ${ }^{3}$, Kristine M. C. Talley ${ }^{4}$, Ryan Haggart ${ }^{5}$, \\ Nidhi Kohli ${ }^{6}$, Badrinath R. Konety ${ }^{7}$, Darryl Mitteldorf ${ }^{8}$, Elizabeth J. Polter ${ }^{1}$, Michael W. Ross ${ }^{2}$, \\ William West ${ }^{9}$, Christopher Wheldon ${ }^{10}$, Morgan Wright ${ }^{1}$
}

${ }^{1}$ Division of Epidemiology and Community Health, School of Public Health, University of Minnesota, Minneapolis, MN, USA; ${ }^{2}$ Department of Family Medicine and Community Health, University of Minnesota Medical School, Minneapolis, MN, USA; ${ }^{3}$ Department of Radiology, Trinity Teleradiology Services, Vancouver, BC, Canada; ${ }^{4}$ Adult and Gerontological Health, University of Minnesota School of Nursing School, Minneapolis, MN, USA; ${ }^{5}$ Department of Urology, University of Minnesota Medical School, Minneapolis, MN, USA; ${ }^{6}$ Department of Educational Psychology, University of Minnesota, Minneapolis, MN, USA; ${ }^{7}$ Department of Urology, Rush Medical College, Chicago, IL, USA; ${ }^{8}$ Malecare Cancer Support, New York, NY, USA; ${ }^{9}$ Department of Writing Studies, University of Minnesota, Minneapolis, MN, USA; ${ }^{10}$ Department of Social and Behavioral Sciences, College of Public Health, Temple University, Philadelphia, PA, USA

Contributions: (I) Conception and design: BRS Rosser, A Kapoor; (II) Administrative support: EJ Polter, M Wright; (III) Provision of study materials or patients: BRS Rosser, D Mitteldorf, EJ Polter; (IV) Collection and assembly of data: BRS Rosser, A Kapoor, EJ Polter, M Wright; (V) Data analysis and interpretation: BRS Rosser, GN Rider, KMC Talley, R Haggart, N Kohli, BR Konety, EJ Polter, MW Ross, W West, M Wright, C Wheldon; (VI) Manuscript writing: All authors; (VII) Final approval of manuscript: All authors.

Correspondence to: B. R. Simon Rosser, PhD, MPH, LP. University of Minnesota, 1300 S. 2nd St. \#300, Minneapolis, MN 55454, USA.

Email: rosser@umn.edu.

\begin{abstract}
In 2016, the NIH designated sexual and gender minorities (SGM) a health disparity population. The next year, the American Society of Clinical Oncology highlighted the need to improve the suboptimal cancer and survivorship care received by SGM populations. There are currently no evidence-based training programs in culturally competent care of prostate cancer patients who are gay, bisexual and/or transgender. In this selective review, we summarize findings from the largest quantitative studies focused on sexual minority prostate cancer survivors and from 65 interviews with NIH staff, clinicians, and cancer clinics in 11 US cities. The report is divided into three parts and uses a question and answer format to address 21 questions relevant to clinicians providing care to SGM prostate cancer patients. First, we identify population-specific issues that are culturally relevant in the care of SGM patients with prostate cancer. While a body of research has emerged on sexual minority prostate cancer patients, the literature on gender minorities is limited to single case reports and inadequate to inform practice. This review covers definitions, population size, cultural and historical context, sexual behavior, population invisibility, sexual orientation and gender identity (SOGI) in the electronic medical record, disparities and evidence of discrimination in treatment provision. The second part focuses on promoting evidence-informed, patient-centered care. This includes current practices in assessing sexual orientation, management of disclosure of sexual orientation, how to address common problems sexual minority men experience post-treatment, common questions sexual minority patients have, management of urinary incontinence, HIV and STI risk during and post-treatment, and sub-groups of sexual minority patients with worse outcomes. It then identifies how male partners differ in prostate cancer support, current research on rehabilitation for sexual minority men, issues in advanced prostate cancer, and things to avoid with minority patients. Finally, we examine the cultural divide between provider and patient, advocating for cultural humility when working with minority patients. Training programs and continuing education can help providers both to become more aware of their own cultural assumptions, informed about health disparities, and able to provide quality care, and to make clinics more welcoming to SGM patients.
\end{abstract}


Keywords: Health disparities; sexual and gender minorities (SGM); prostatic cancer

Submitted Jun 30, 2020. Accepted for publication Nov 19, 2020.

doi: $10.21037 /$ tau-20-1052

View this article at: http://dx.doi.org/10.21037/tau-20-1052

This article is divided into three parts. First, we summarize the emerging literature on research on SGM prostate cancer survivors to answer the question how do SGM patients differ from majority (i.e., cisgender, exclusively heterosexual) patients. Secondly, we describe how clinical care can be tailored to improve care for these populations. In the final part, we address how training programs and continuing education can help providers to improve care by promoting cultural humility in providing care to this population.

\section{Summary of methods}

Prostate cancer in gay and bisexual men is a new area of research beginning in 2000. Currently, the entire literature is around 100 published papers, although most of these are qualitative studies, commentaries, or single case reports. Several excellent reviews of cancer in $\operatorname{SGM}(1,2)$ and specifically of prostate cancer in SM $(3,4)$ have summarized key findings, while a meta-synthesis of the qualitative studies on prostate cancer in gay and bisexual men was recently published (5).

As the American Society of Clinical Oncology noted recently, limited research is being conducted to assess the needs and establish standards of care for SGM patients with cancer and cancer survivor populations ... [Further], concern has been expressed that the SGM community experiences a heightened perception of more cancer and worse outcomes, even if this is not always concordant with available epidemiologic data (6).

To address ASCO's concern, the next step is to review the quantitative findings across studies of prostate cancer in SGM. Globally, there have only been seven quantitative studies of this population to date (7-14). For this selective review, three were deemed of sufficient size, statistical rigor, and used similar, validated measures [including the Expanded Prostate Cancer index Composite or EPIC, and the Short Form (SF) Health Survey] that we can compare results across studies (7-9). By contrast, the literature on prostate cancer in GM is limited to single case study reports and inadequate to provide an empirical-base to inform clinical practice specific to gender minority patients (15-19).
The studies are as follows:

In 2010, Hart et al. $(7,20)$ conducted a study of 92 selfidentified gay and bisexual men diagnosed with prostate cancer within the prior four years. Participants had to be living in North America and were recruited from list-serves targeting prostate cancer survivors and from community centers, support groups, and local media advertising in two cities (Houston and Toronto).

Ussher et al. $(8,21,22)$ compared the responses of 124 SM with 225 heterosexual men diagnosed with prostate cancer. SM participants were recruited within Australia from urology and primary care clinics, cancer support groups and gay community organizations, and internationally from SM social media and listservs targeting prostate cancer survivors. Most were from Australia, the United States or the United Kingdom. The heterosexual comparison group was recruited from an Australian cancer research volunteer database.

Restore- 1 was a mixed methods study conducted by our team in 2015-2016 (9). First, we conducted in-depth qualitative telephone interviews with 30 prostate cancer patients and nine partners or caregivers (23-25). Then, we conducted an online quantitative survey with 192 gay and bisexual and one transgender prostate cancer patients/ survivors living in North America (26-32). Participants were recruited from the largest online cancer support network providing groups for SGM prostate cancer patients.

A fourth study, conducted by Allensworth-Davies et al. (13) in 2011, recruited 111 gay-identified prostate cancer survivors in the US. This study was of sufficient size and used similar measures to those above to be included. However, they did not statistically compare their results to published norms for heterosexual patients and so the results cannot be included.

In addition, three other studies conducted by our team add findings to the evidence base. Restore-2 [2017-2022] is a randomized controlled trial and the largest study to date, currently in progress, testing the effects of an online comprehensive sexual and urinary rehabilitation program tailored for SGM in 401 US gay and bisexual prostate cancer patients (33). Participants had to have been treated 
for prostate cancer and were recruited both online and from clinics. To answer the question how SGM patients became "invisible" in prostate cancer research and clinical practice, in 2018, we completed 65 key informant interviews with NIH staff, clinicians and urology clinics in 11 US cities. Finally, to assess the acceptability and feasibility of adding SOGI data to the patient electronic medical record, we conducted a study with 101 urology and 104 oncology patients, then assessed data on the first 450 urology and 103 oncology patients to answer the question in their EMR (34).

\section{Part 1: understanding the research on SM prostate cancer survivors}

\section{What is the definition of a sexual or gender minority (SGM)?}

Recently, the National Institutes of Health designated the term sexual minorities (SM) to denote lesbian-, gayand bisexual-identified individuals, plus those who do not selfidentify but who experience same-sex sexual attractions and/or behavior (35). Gender minority (GM) denotes those who identify as transgender or non-binary, or with a gender that does not align with social and cultural expectations for their birth-assigned sex (e.g., agender, gender fluid, genderqueer) $(35,36)$. The terms contrast with the majority population, which denotes cisgender, exclusively heterosexualidentified and oriented persons. NIH recognizes SGM as health disparity populations, meaning that compared with cisgender, heterosexual patients, SGM patients, on average, have higher burden of illness, poorer experiences in treatment, and worse outcomes $(35,37)$.

\section{What is the size of the SGM population?}

In nationally representative surveys, SGM adults comprise $4.5 \%$ or 11.5 million of the 255 million adults living in the US (38). Estimates vary across surveys (39), but appear to be increasing over time (40). These estimates vary by subgroup: $5.1 \%$ of women identify as lesbian, gay or bisexual; $3.9 \%$ of men identify as gay or bisexual; and $0.6 \%$ of adults identify as transgender or gender non-binary (40). (Transgender and non-binary people may identify as lesbian, gay, bisexual or heterosexual). This contrasts with $95.5 \%$ US adults who identify as cisgender and exclusively heterosexual. But within SGM, there are large gender and age differences. Regarding gender, SM men (42\%) are disproportionately under-represented relative to SM women (58\%); and across age, older persons are significantly under-represented relative to both younger cohorts of SM and similar aged cohorts of cisgender, heterosexual peers (38). Only $10 \%$ of SGM are aged 65 years or older compared with $19 \%$ of heterosexuals in this age group (38). These differences likely reflect higher mortality in SM male and transgender older cohorts due to HIV/AIDS, suicide, and other factors; and possibly that older cohorts may be less likely to self-identify as LGBT. As HIV has become a manageable chronic illness and homosexuality less stigmatized, the proportion of SGM prostate cancer patients in the next decades should increase from the current level of about $2 \%$ to around $4 \%$. This suggests that of the 3.1 million men diagnosed with prostate cancer in the US (as of 2017) (41), about 63,000 are sexual minorities.

\section{How are SGM different from other patients?}

The LGBT community is highly diverse so it is important to emphasize that no one datum applies to all. Like other minorities, SGM experience increased stress, discrimination, violence and isolation (42). All these impact health, increasing disorders of anxiety, depression, suicidality, and internalized homonegativity or transnegativity (i.e., a negative self-concept as damaged, flawed or less than others because of one's homosexual attractions, behavior, orientation or gender identity). Minority stress also predicts increased risk of alcohol, drug, nicotine use, abuse and dependence as well as using sex as a psychological coping strategy (43).

Appreciating the life histories of those in the age cohort for prostate cancer provides critical context for care. Most older SGM lacked positive role models as children, and many lived in states that criminalized and stigmatized homosexual behavior and gender non-conforming presentations. Many faced rejection by one or more family members. Almost all endured social stigma, condemnation from their religions-of-origin, insecurity in employment, and the threat of violence when in public. From 1981, this cohort survived the HIV/AIDS pandemic, which killed an estimated 600,000-750,000 SGM in the US. Currently, about $20 \%$ of older SM men and transwomen in the US are living with HIV. So, for many SGM patients, their lifelong experience of trauma and discrimination psychologically changes the way they experience a prostate cancer diagnosis and treatment, and their trust in the medical community.

Given what they endured, older SGM individuals may 
also be described as resilient survivors. Being less likely to have close ties to family-of-origin, to have children, or to be in a long-term relationship, many constructed families-of-choice, relying on internal resources and a network of friends for mutual support and love (44-46). Given the social stigma of homonegativity and/or gender non-conformity, coming out for many was a pivotal act of individual courage and survival, which in turn built assertiveness (including with health providers). Collectively, this cohort has advocated and experienced huge social change, from being illegal outcasts to having their rights and relationships recognized. For GM, advances in the understanding of gender diversity as non-pathological and in gender affirming surgeries have provided new options to be themselves. SGM share with other minorities the experience of being different from the majority culture. While some may choose to assimilate, "out" SGM individuals are more likely to have rejected multiple social norms, to question rules and challenge authority, and to assume they need to be an advocate for themselves and others in their healthcare.

\section{What should a provider know about same-sex sexual behavior, specific to SM men with prostate cancer, in order to provide good care?}

SGM individuals are as diverse as heterosexual people are in their sexual behavior. So, to treat the sexual effects of prostate cancer, a provider needs to ask about sexual practices. Because same-sex sexual behavior has multiple options and roles-in-sex, it requires a detailed assessment if the clinician is to understand the patient's situation and concerns.

As a group compared to older heterosexual men, older SGM are more likely to be single (47), sexual (both with self and others), to have more lifetime partners, and to view their sexual health as a core component of their identity $(48,49)$. For the half of SM men in relationships $(50)$, some couples are monogamous, while others have relationships open to other partners (usually with rules about how this is handled) (51). For SM men in heterosexual relationships, they may have similar understandings with wives and other female partners about their sex with male partners (52). SGM individuals who are closeted, by definition, typically do not reveal their sexual liaisons to others.

The most common sexual behaviors between gay, bisexual and other men who have sex with men are masturbation (both with and without anal stimulation), oral sex, and anal sex, followed by rimming (i.e., analingus) (53). While some men may prefer being both the receptive and the insertive partner in sex (called being "Versatile"), other men discover they prefer being the insertive partner (termed a "Top") or the receptive partner (termed a "Bottom") (54). In this age cohort, and in some racial/ethnic minorities, being the receptive partner still carries additional stigma, making Bottoms less likely to admit to being the receptive partner and to ask relevant questions about the effects of treatment on their behavior $(55,56)$.

There are other common practices to be aware of when providing prostate cancer treatment to SGM patients. Use of nitrites (termed "poppers") is very common to enhance sexual sensation during sex and must be assessed before prescribing PDE-5-inhibitors. Ejaculate is especially important to some SM men (57). These patients report a profound sense of loss and chronic depression following prostate cancer treatment specific to loss of ejaculate (23). Oral sex is a common practice particularly with new partners and in casual dating, so concerns about urinary incontinence and its effects on sex and dating should be anticipated. While most SGM know as little as heterosexual men about the prostate as an organ, gay pornography and culture highlights prostate stimulation as the ultimate source of sexual pleasure (e.g., through digital stimulation during masturbation and penile stimulation in receptive anal sex). Most SGM use a water-based or silicone-based lubricant when having anal sex. In addition, about half of SM report enema use before receptive sex; with the most common products used being commercially available enemas, water, and water with salt, soap or antibacterials added (58). A minority of SGM engage in brachioproctic (i.e., "fisting") sex practices, where a partner's hand, fist and/ or arm is inserted into the anus.

\section{Why are SGM prostate cancer patients so "invisible"?}

In 2005, in summarizing the literature at that time, Perlman and Drescher concluded, "If prostate cancer, in general, is off most people's radar screen, then gay men with prostate cancer are a truly invisible species" (59). To answer the question how SM patients became or remain "invisible" in clinics, we interviewed 65 key informant NIH staff, clinicians and urologists in 11 US cities. Our social ecological analysis found barriers at every level (see Figure 1). This suggests that to improve clinical care and outcomes for SM patients, physician education and systemic interventions at the clinic level are needed. For clinicians, 


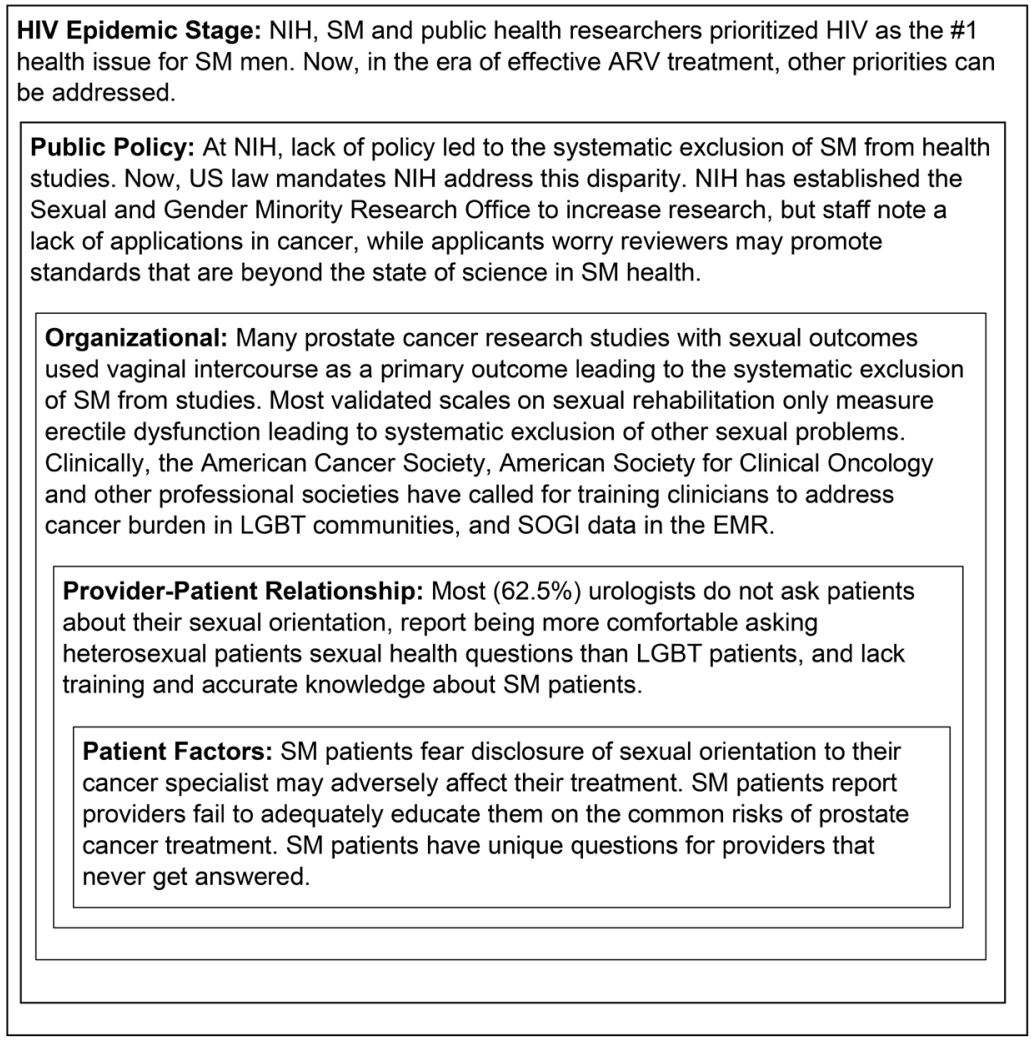

Figure 1 The Modified Socioecological Model* applied to making prostate cancer in sexual minority men more visible in research and clinical practice. *, The Modified Social Ecological Model is adapted from: Baral S, Logie CH, Grosso A, et al. Modified social ecological model: A tool to guide the assessment of the risks and risk contexts of HIV epidemics. BMC Public Health 2013;13:482.

we highlight a recent survey of 112 urologists in the US (60). Most said they do not ask patients their sexual orientation, setting up a "don't ask; don't tell” dynamic. They stated they were significantly more comfortable discussing sex with heterosexual patients than with SM patients, that they lack accurate knowledge about SM patients, and reported receiving less than 5 hours of education about this population in medical school, residency, or through continuing education. Most felt more training is needed (60).

The absence of transgender prostate cancer patients and studies likely reflects the protective effects of estrogen and orchidectomy on the development of prostate cancer. And it also reflects significant mortality at younger ages from HIV, suicide and violence. Structural factors may exacerbate the perception of population invisibility caused by clinic collection of gender data using binary measures; less access to screening and care; possibly less patient willingness to acknowledge and present with a "male-specific" health concern; and lack of clinical options where GM can receive welcoming, comprehensive clinical care. Research on prostate cancer in GM is needed.

\section{What is the easiest way to screen for sexual orientation and gender identity (SOGI)?}

To facilitate research on SGM disparities, NIH has encouraged clinics to routinely collect SOGI in the patient electronic record (61). Some clinics have resisted this citing acceptability concerns about their conservative, cisgender, heterosexual patients being offended by being asked about their sexual orientation or seeing a non-binary question on gender. Recently, we assessed the acceptability and feasibility of collecting SOGI data at a Midwestern oncology and urology clinic (see Figure 2) (34). Over 90\% patients reported the response options were understandable and easy to answer, and over $95 \%$ answered it when placed in their EMR. Adding SOGI data to the EMR appears to 
1. Sexual Orientation Question:

Do you think of yourself as:

Lesbian, gay or homosexual

Bisexual

Straight or heterosexual

Something else, please specify:

Do not know

Choose not to disclose

\title{
2. Gender Identity Question:
}

Sex assigned at birth:

Female

Male

Choose not to disclose

\author{
Gender identity \\ Female \\ Male \\ Transgender (female to male) \\ Transgender (male to female) \\ Other, please specify: \\ Choose not to disclose
}

Source: Cahill S, Singal R, Grasso C, King D, Mayer K, Baker K, Makadon H. Do ask, do tell: High levels of acceptability by patients of routine collection of sexual orientation and gender identity data in four diverse American community health centers. PloS One. 2014;9(9):e107104.

Figure 2 Standardized Sexual and Gender Identity Questions for the Patient Electronic Medical Record.

be a simple and efficient way to overcome the "don't ask" practices of most urologists, to facilitate research, and to increase visibility of these patient groups.

\section{What should a provider know about sexual minorities and cancer?}

SM men may have a higher lifetime incidence of cancer than heterosexual men (62), and higher rates of some cancers (e.g., anal, lung, colorectal, oropharyngeal, HIVrelated) (63). At every stage of the cancer continuum, SM have higher rates of risk factors (e.g., sex, STIs, alcohol, nicotine, tanning, stress) (64), lower insurance rates (65), less screening (66), less familial and social support $(26,67)$, later diagnosis (61), more distress (68), unique challenges (such as disclosure of sexual orientation) (69), less culturally competent care (70), and worse health (62) and quality of life outcomes $(8,71)$. We caution that most of these results are based on small sample sizes and need further study.

\section{What should a provider know about gender minorities and cancer?}

GM also experience increased cancer risk factors (72-76), stigma in health care provision (77-81), victimization and discrimination (82-84), and resulting mental health challenges $(85,86)$. Malignancies related to hormone use are rare (87-89); indeed, hormone use and gender affirming surgery may be protective for some cancers including prostate cancer while increasing risk for others (e.g., breast) (90). In cancer care, GM face added challenges of GM disclosure, less or inadequate screening $(91,92)$, fewer 
cancer specialists comfortable in providing comprehensive care (93), and lack of protocols for GM-specific care. Transgender women (i.e., a person who identifies as a woman and was assigned male at birth) are the highest risk population for HIV and other sexually transmissible infections (75). In cancer outcomes, transgender women have higher mortality due to non-treatment related causes (i.e., suicide, HIV, and drug abuse) (89).

\section{What are the key differences in outcomes between SM and majority patients?}

In both Restore-1 and Hart et al., studies of North American SM prostate cancer patients, SM patients had significantly worse urinary function and hormonal function and bother but better sexual function and bother scores than published norms for heterosexual patients $(7,9)$, [Ussher et al. only assessed sexual function but also found it better than their heterosexual controls (8), while Allensworth-Davies scores mirror the other studies as well (13)]. We think the poorer urinary scores may reflect SM's arousal incontinence and climacturia concerns during oral sex. The better sexual functioning outcomes may be some combination of the following factors. Sex appears more central to SM men's identities and behavior, which could result in more commitment and openness to rehabilitation. Behaviorally, SM have better physical health and may masturbate more following treatment aiding erectile recovery. A greater percentage of heterosexual men may be in relationships that are no longer sexual and may be less motivated for rehabilitation. SM patients are also more likely to engage in novel accommodation strategies that most heterosexual couples rarely adopt. These include use of sex and hookup sites to meet new partners, experimenting with changes in role-in-sex, and in opening up their relationship to other partners. Alternatively, it simply may be a research artifact. Given EPIC was normed prior to the use of PDE5-i drugs to treat erectile dysfunction (ED), the sexual scores of heterosexual men may have improved as well since the scale was normed in 2001.

Hart et al. and Restore-1 used the Medical Outcomes Study Short-Form (SF-36 and SF-12, respectively). Both found SM scored worse on mental health than norms for heterosexual prostate cancer patients $(7,9)$. Ussher et al., using other measures, also found worse mental health on multiple scales. While Hart et al. and Ussher et al. found no difference in physical health (but were smaller studies),
Restore-1 found SM had significantly better physical health than norms for heterosexual men. The worse mental health in all three studies is consistent with the broader literature that SM men have worse mental health secondary to minority stress, experiences of discrimination, and trauma (94). The better physical health in Restore- 1 is consistent with stereotypes of this population being more likely to exercise, to go to gyms, and to be committed to staying in shape.

\section{What about differences in experience of treatment?}

There are several differences to consider when treating SM patients. First, about half of SM prostate cancer patients are single and many lack the social support their heterosexual peers have. Hence, they are much more likely to undergo treatment, alone; and more likely to only discuss their prostate cancer with their provider. Second, a common experience across racial, ethnic, sexual and gender minorities is the need to assess whether the provider is competent in providing care to that minority compared to majority patients who may simply assume, usually rightly, that treatment is already tailored or optimized for men like them. Third, especially for SM men who are out to their primary healthcare providers, and for those HIV positive, they are used to and may insist upon shared decisionmaking in their health care. To more authoritarian providers and conservative clinic systems, this may make them seem more demanding and less compliant. Fourth, case reports affirm the importance of SGM talking to other SGM about their cancer (57), however, general prostate cancer support groups may adversely affect SGM if they feel alienated from discussing their sexual concerns (95). Fifth, a significant minority of SM patients report poorer care. In Restore-1, about $20 \%$ of the sample said they received poorer prostate cancer care than other patients. Of these, $43 \%$ reported that the provider did not listen, $25 \%$ that they were talked down to, $19 \%$ that the provider acted superior, and $10 \%$ that the provider appeared afraid of them. Almost half ( $9 \%$ of the $20 \%$ ) attributed the poorer care they received to their sexual orientation. The remainder wrote in comments attributing it to going in as a gay couple, being gender non-conforming, or to the provider being arrogant, a jerk, or too pushed for time (unpublished data).

The high-level summary from these statistics is that SGM patients, in common with other minorities, report higher rates of risk factors, greater barriers to care, poorer 
Table 1 Clinical questions to ask prostate cancer patients at assessment

Prostate cancer treatment can have effects on your sex life. To provide you with the best care, l'd like to ask you a few questions

1. Do you have a primary partner? If yes, then invite the patient to include their partner in any future sessions to address the partner's concerns. If the patient does not specify a gender of their partner, then ask a prompt to clarify gender

2. Do you have sex with men, women, or both?*

a. Women (only). Direct the discussion towards surgery or radiation options and consider interventions that enhance erectile ability (e.g., erectile enhancing drugs, vacuum pump, penile injections). Focus follow-up discussions on erectile ability

b. Men, or both. If the patient reports he has sex with men (or both) then continue

3. [If sex with men, or both]? In sex, are you more a top, bottom, or versatile?

a. If answers "top" [insertive partner]: Direct the discussion more towards surgery and radiation outcomes and consider interventions that enhance erectile ability (e.g., erectile enhancing drugs, vacuum pump, penile injections). Focus follow-up discussions on erectile ability

b. If answers "bottom" [receptive partner]: Direct the discussion more towards surgery (since $20 \%$ of radiation patients have radiated bowel, making resumption of receptive sex, impossible), provide information about how long to refrain from receptive stimulation postintervention. Focus follow-up discussion on ability to engage in receptive anal sex asking about any loss of pleasure or change in pain

c. If answers "versatile" [both insertive and receptive partner]: Direct the discussion more towards surgery (since $20 \%$ of radiation patients have radiated bowel, making resumption of receptive sex, impossible; while both surgery and radiation have similar outcomes on ED at two years' follow-up), provide information about how long to refrain from receptive stimulation, post-intervention. Encourage the patient to think through how easy it would be for them to shift roles in sex if they could only be in in one role, and focus follow-up discussion on both roles in sex

4. How often do you use poppers? (Popper [nitrite] use is very common among gay and bisexual men in this age cohort to enhance sensations of pleasure/intensity in sex and to decrease any pain)

a. Never

b. Anything more than never. Prior to prescribing any erectile enhancing medication, explain their potentially lethal interaction with poppers

*, dependent on the time the clinician has to conduct the interview and the depth of information sought, an alternative format for asking this question, which is more inclusive, is to ask, "And what is the gender or genders of your partners?" This allows patients to identify their partner(s) as male, female, transgender, non-binary or any combination of these. A third option is simply to ask about behaviors by body parts (e.g., penis-vaginal sex, penis-anal sex). Source: Rosser BRS, Konety BR, Mitteldorf D, et al. What gay and bisexual men treated for prostate cancer are offered and attempt as sexual rehabilitation for prostate cancer: Quantitative results from the Restore study with implications for clinicians. Urol Pract 2018;5:187-91.

experience in treatment, and worse outcomes. So, what can providers do to improve the treatment experience for their SGM patients? This next part focuses on promoting evidence-based, culturally responsive care.

\section{Part 2: Tailoring the clinical care of SGM prostate cancer patients}

\section{What is current best practice for assessing sexual orientation?}

Providers can sometimes forget how disorienting and frightening a referral to a urology or oncology clinic can be for patients. For racial minority SGM, it may be even more so. Patients may feel intimidated by the specialists and fearful of the diagnosis. They also may not know what questions to ask or how much information to disclose. So, patients normally take their cue from their provider. Unfortunately, most providers $(63 \%$ urologists and $74 \%$ oncologists) do not ask about sexual orientation at first assessment. This may explain our finding in Restore-1, that while $77 \%$ of participants had disclosed their sexual orientation to their primary provider, significantly less were "out" to their urologist (60\%), surgeon $(59 \%)$, or oncologist (56\%). At first assessment, we recommend providers ask all patients (who have not disclosed their sexual orientation) four screening questions (see Table 1). 
Table 2 Patient reports of the common problems they experienced post-treatment and whether the provider discussed the problem prior to treatment (Restore-1 Quantitative Survey; N=193 Gay and Bisexual Prostate Cancer Patients)

\begin{tabular}{|c|c|c|}
\hline Sexual concern & Discussed prior to treatment & Experienced* \\
\hline Loss of ejaculate (semen or cum) & $71.0 \%$ & $95.3 \%$ \\
\hline Erection difficulties & $74.1 \%$ & $91.1 \%$ \\
\hline Change in your sense of orgasm & $24.4 \%$ & $88.4 \%$ \\
\hline Changes to the penis (size, shape) & $23.3 \%$ & $66.8 \%$ \\
\hline Urinary problems (bladder control) not related to sex & $74.6 \%$ & $64.9 \%$ \\
\hline Loss of sexual desire/libido (interest in sex) & $37.8 \%$ & $59.4 \%$ \\
\hline Urinary problems during sex or at orgasm & $29.5 \%$ & $49.1 \%$ \\
\hline Increased pain or problems during receptive anal sex & $3.6 \%$ & $13.2 \%$ \\
\hline For fathering children the need to sperm bank prior to treatment & $22.3 \%$ & $10.5 \%$ \\
\hline
\end{tabular}

*, \# reporting problem/\# reporting and reporting not experiencing problem (excludes don't recall and refuse to answer). Source: Rosser BRS, Konety BR, Mitteldorf D, et al. What gay and bisexual men treated for prostate cancer are offered and attempt as sexual rehabilitation for prostate cancer: Quantitative results from the Restore study with implications for clinicians. Urol Pract 2018;5:187-91.

\section{How should sexual orientation disclosure be handled, clinically?}

Sometimes patients may disclose their sexual orientation or gender identity to their provider. The provider should recognize that such disclosure is a highly important, personal detail to the patient. The appropriate response is to affirm the disclosure, for example by saying, "Thank you for letting me know. That's really helpful information for when we discuss the effects of treatment." Such a response invites greater disclosure and trust in the therapeutic relationship, and provides permission to both the patient and the provider to raise questions about the impact of treatment on the patient's sexual behavior.

In our Restore-1 interviews, SM patients reported four key strategies around sexual orientation disclosure to their prostate cancer specialists: (I) to out themselves, by explicitly referring to themselves as gay or bisexual (or recording it in the EMR); (II) to evaluate the specialist on demographic or other criteria to decide whether it is safe to disclose; (III) to assume the specialist already knows or can infer their sexual orientation from demographics; and (IV) to elect not to disclose (96). The response of the provider is critical. In our qualitative interviews, when the urologist or oncologist treated it sensitively, disclosure appeared to increase patient trust in the physician-patient relationship (96). However, when the physician discounted or appeared uncomfortable with the disclosure, the patient terminated treatment (96). Asking about SOGI in a non-judgmental and sensitive way can inform cancer care and is the first step to building trust with SGM patients.

\section{What to discuss when informing patients about the sexual effects of treatment?}

Like other patients, when SGM prostate cancer patients are provided with relevant information on the effects of treatment, they can prepare and adjust to the effects of treatment. But when they experience effects without being forewarned, they report lasting resentment at being inadequately informed (23). In Restore-1, we asked SM prostate cancer patients what were the most common problems they experienced, post-treatment, and whether their provider had discussed these with them prior to treatment (see Table 2). Most patients recalled that providers discussed loss of ejaculate, erectile difficulties and 
Table 3 Common sexual questions and suggested answers for sexual minority prostate cancer patients

1. Which prostate cancer treatment will have the least effects, sexually? That depends on what sexual behaviors are most important for you. For insertive sex, both radiation and surgery with nerve sparing have similar effects on your ability to get erections (at two years' follow-up). For receptive sex, radiation therapy is not recommended as about 1-in-5 patients will have radiated bowel and will not be able to ever engage in receptive sex, post treatment

2. How long should I wait after my biopsy before anal stimulation? The time you should wait after a biopsy varies and depends on what is being inserted into the anus. For insertion of a finger, gentle stimulation as soon as you feel comfortable is fine. For insertion of a penis or dildo of penile width, wait about two weeks to allow sufficient time for healing. Start with finger insertion before attempting insertion with a penis or dildo. For insertion of an arm or fist, most doctors would counsel you to avoid fisting if diagnosed with prostate cancer. There is no science to guide this recommendation, but it's prudent. If you decide to be fisted, wait a minimum of at least six weeks after a biopsy

3. How long after a radical prostatectomy should I wait before resuming anal stimulation? How much healing time is needed can depend on the surgical technique, and on what is being inserted. Here is a general guide for the average patient. For insertion of a finger, wait at least six weeks after surgery. This is to allow time for the surgical site to heal. Use your own finger at first (after trimming nails) and stop if you feel any discomfort. Gradually practice and once comfortable, allow a partner to stimulate you anally with their finger. For insertion of a penis (or a dildo of average penis width), wait a minimum of eight weeks. Before resuming sex with someone else, practice on yourself by using a dildo, vibrator or butt plug. For fisting: after a radical prostatectomy, if the healing is complete, then there should be no additional risk from fisting. But (I) wait a minimum of 9-12 weeks after surgery, and (II) speak with your urologist first in case s/he is aware of medical limitations

4. How long after being treated with radiation should I wait before resuming anal stimulation? For patients treated with radiation, because the radiation has lasting effects on weakening the anal lining, the recommendation is to avoid anal stimulation

Source: Rosser BRS, et al. Recovery after prostate cancer: A comprehensive guide to rehabilitation for gay and bisexual men. (An online randomized controlled trial currently in progress).

urinary problems with them. But most patients reported providers failed to discuss with them other very common challenges. These include in their sense of orgasm, loss of sexual confidence, changes to the length, color and/ or curvature of their penises, loss of desire, urinary problems in sex, and loss of pleasure or the ability to orgasm in receptive anal sex. By briefly covering these with patients, providers empower patients to make more informed choices in their treatment course, prepare them for what to expect, and encourage them to raise concerns if they experience them.

\section{What are the most common questions SGM have that they may be reluctant to ask?}

SGM patients have a set of common questions about the effects of biopsies and treatment, which they do not normally ask out of fear of embarrassment and stigma. Providers can improve outcomes by directly addressing these as part of post-biopsy and post-surgical or radiation care. Currently, there are no standard answers to these questions. So, we list a set of questions with suggested answers (taken from the Restore-2 rehabilitation curriculum; see Table 3).

\section{What should a clinician address about urinary incontinence?}

When treating SGM, it is a mistake to only focus on the sexual effects of treatment. Urinary incontinence is a common, yet underreported and undertreated effect as well. The prevalence of urinary incontinence in SM treated for prostate cancer is probably similar to that in the general population of prostate cancer survivors (97). In two studies, we have evidence of greater urinary symptoms and bother in SGM than majority patients, although the reasons for this are unclear $(7,9,32)$. In Restore-1, about one third of SM reported leaking urine at least daily, and over half reported some urinary incontinence (32). Types of prostate cancer treatment were associated with different types of urinary incontinence $(98,99)$. SM treated with surgery (with or without radiation) experienced greater severity of urinary incontinence and more symptoms of stress urinary incontinence and insensible urinary incontinence than SM treated with radiation only. SM treated with radiation only experienced more symptoms of urge incontinence. In SM, urinary incontinence severity was associated with obesity, poor self-reported health, and an increased number of comorbidities but not with time since diagnosis. 
Among men who seek treatment for urinary incontinence, the SM in our Restore-2 study reported greater severity of urinary incontinence symptoms and bother than the general population of men enrolled in the large Prostate Testing for Cancer and Treatment (ProtecT) trial (98). Furthermore, in SM the degree of bother with urinary incontinence was associated with worse physical quality of life.

Clinicians should screen SM prostate cancer survivors for urinary incontinence. The ICIQ is a short 4-item screener that assesses the severity and type of urinary incontinence and the degree of bother this symptom causes (100). Treatments for SM patients treated surgically should focus on treatments for stress urinary incontinence and insensible urinary incontinence, while those for SM patients treated with radiation should focus on treatments for urgency urinary incontinence.

\section{What are the other clinical issues a provider should prioritize?}

Addressing HIV/STI transmission as part of prostate cancer treatment is a priority. In both Restore- 1 and -2 studies, we have evidence that $5-10 \%$ patients became infected with HIV or other sexually transmissible infections, post-treatment. More research is needed to confirm whether HIV/STI transmission is incidental to or a direct consequence of prostate cancer treatment. In the interim, to avoid harm, we advise addressing HIV/STI risk with all SGM prostate cancer patients.

This is not as simple as recommending to just use a condom. At least five factors may be contributing to HIV/ STI acquisition during and after prostate cancer treatment. First, $85 \%$ of Restore- 1 participants rated their erections as inadequate for insertive anal sex, with $61 \%$ reporting soft erections as their reason for non-condom use (9). Second, to accommodate erectile dysfunction caused by treatment, many Tops experiment with being the receptive partner. This increases their HIV risk, twenty-fold. Third, most $(57 \%)$ receptive partners reported one or more occasions of painful sex. We estimate one-in-three receptive partners have anodyspareunia, post treatment; approximately double the rate, pre-treatment (9). Only 29 percent of receptive partners reported their partner used a condom (9). More research is needed to confirm whether pain in receptive sex may lead receptive partners to have unprotected anal sex. Fourth, in qualitative interviews, several men in couples stated they opened up their relationship either because the insertive partner had ED or the receptive partner had anodyspareunia from treatment (24). Fifth, some men who may have preferred oral sex, pre-treatment, may switch to anal sex to avoid embarrassment about arousal incontinence or climacturia.

Providers need to recognize SM men and transgender women are at highest risk for HIV/STIs, explicitly ask about changes in HIV/STI risk as part of comprehensive care, and work with the patient to devise a realistic plan to reduce HIV/STI transmission. Condom use should be addressed when discussing erectile dysfunction, along with alternatives such as treatment-as-prevention (i.e., TasP, if HIV-positive) or pre-exposure prophylaxis (i.e., PrEP, if HIV-negative).

\section{Which groups of SGM are at greater risk of poorer outcomes from prostate cancer treatment?}

Four subgroups of SGM appear most at risk of poorer outcomes. First, HIV-positive patients score worse on EPIC sexual and urinary subscales compared to HIV-negative patients, post-treatment (28). Second, when we compared Restore-1 participants by age, similar to heterosexual patients, the older cohorts scored worse on SF-12 scores for mental health and physical health $(\mathrm{P}<0.05)$. But in addition, the older participants were less likely to be "out" about their sexual orientation (101). Older cohorts may be less likely to disclose their sexual orientation to their specialist (unless asked), are more at risk of being assumed to be celibate, and more likely to have less social support. Third, as compared to gay-identified men, bisexual-identified men have worse outcomes across a variety of health metrics, although research on prostate cancer patient outcomes by orientation are lacking (but are planned). Fourth, SM prostate cancer patients rely on partners/husbands, gay friends or chosen family and men from SGM prostate cancer support groups for informational and emotional social support. In Restore-1, patients with less social support had significantly worse hormone, sexual and bowel bother symptoms, and worse mental health (26).

\section{What do we know about male partners of prostate cancer patients?}

This is a very under-researched area restricted to qualitative interviews and the Restore-1 data. But we can infer some differences. Demographically, unlike most heterosexual couples where wives are typically younger than their husbands, SM patients' partners may be as old or older and 
not able to provide care. While wives/female partners of heterosexual patients tend to focus on survival $(102,103)$, husbands/male partners appear equally concerned about the sexual effects of treatment (26). Because SM men are men, both patient and partner may be more likely to handle the patient's diagnosis alone (104). And because SM men are not well educated about prostate cancer, their partners may fear prostate cancer is sexually transmissible. For patients who are the receptive partner and undergoing radiation, the insertive partner(s) may have additional questions about possible risk of radiation (e.g., through anal sex).

\section{How is rebabilitation different for SGM men compared to other patients?}

Except for the Restore-2 study currently in progress, there has been only one intervention study, a pilot study of bicalutamide in Romania which reported worse sexual functioning in SM compared to majority patients (10). Currently, we do not know if sexual rehabilitation after prostate cancer treatment improves functioning in SGM, but we warn it is a mistake to simply generalize findings from heterosexual studies. For example, with erectile concerns, anal sex requires greater rigidity than vaginal sex, which implies rehabilitation should be more challenging. And we are testing a protocol to study if a combination of sildenafil, vacuum pump, masturbation and gay-tailored education can improve erectile function sufficient for anal sex. In addition, we have protocols in trial to test the effects of anal dilators on anodyspareunia, and on a combination of pelvic floor and sexual exercises to address urinary incontinence during sex.

\section{What about management of SGM with advanced prostate cancer in SGM?}

We lack studies of SGM with advanced prostate cancer that address their unique considerations. From the broader literature, we know SGM facing end-of-life and assisted living have additional challenges. SGM are significantly less likely than majority patients to have family members to provide care $(105,106)$, and more likely to believe they will need residential long-term services and supports (107). Yet, SGM older adults are 5 times less likely to access health care and social services $(108,109)$. Recent exposés have documented the risk of violence, abuse and discrimination toward SGM in care facilities (110). Most (81\%) SGM older adults fear entering nursing homes or assisted living because of potential discrimination (111). Many SM in this situation end up retreating to the closet out of fear for their safety, and concerns of rejection (112).

\section{What are some things to avoid doing or saying with SM patients?}

All patients want to be treated with respect, to be listened to, to have their questions answered, and their concerns validated. SGM patients are no different. Avoid assuming a patient is heterosexual or is the insertive partner in sexual relationships. In qualitative interviews, SM patients in the Restore-1 study had the strongest negative reaction when providers assumed they were heterosexual (especially after being told otherwise), or assumed that the patient was the insertive partner when engaging in sex (23). Avoid minimizing or dismissing the patient's reality. Providers who denied their patient's reality (e.g., changes to the penis), who minimized the effects of treatment, and/or whose verbal and non-verbal reactions to sexual orientation disclosure were hostile, were disdained (23). Similarly, GM patients object to gender and sexuality assumptions being made by providers. When unclear, the provider should simply ask what pronouns the patient prefers, and what gender(s) do they identify as. At the clinic level, it is important to train the entire team as sometimes micro-aggressions and discrimination may occur before the patient even sees the physician. It is important to avoid heteronormativity in clinic environments, patient education materials, and advertising. SM patients report negative reactions to clinic advertising and environments that are heteronormative and exclusionary (113). This includes "man cave", "sportsoriented," and other machismo-themed waiting rooms and advertising, intake forms that describe a spouse as female or gender as dichotic, screening forms and questions that define sexual functioning as erections or vaginal penetration (or sex as reproduction), and patient education that omits any mention or images of SGM.

\section{Part 3. Addressing a cultural divide}

One of the most memorable comments from our key informant study, was from a senior urologist who said, "I don't get why you need to study this [population]. After all, I treat all my patients the same." He meant that he strove to obtain the best surgical outcomes for every patient. But the 
statement is revealing in three aspects. First, it is fallacious in its assumption that treating all patients the same leads to equal outcomes. It does not for racial, ethnic, sexual and/ or gender minority patients, as the literature on health disparities attests. Second, it implies that all the provider needs to be is a good technologist. When the patient (or patient group) is left feeling discriminated against, with poorer outcomes, then a strong argument can be made that the provider and health system failed this patient. Third, it ignores the presence of implicit bias that can adversely impact patient-provider education, patient satisfaction, and patient outcomes.

To provide optimal care, providers need to be aware of their own personal biases, cultural assumptions, and the need for cultural humility in providing care to SGM patients. Cultural humility requires "a lifelong commitment to self-reflection and self-critique, to redressing the power imbalances in the patient-physician dynamic, and to developing mutually beneficial and nonpaternalistic clinical and advocacy partnerships with communities on behalf of individuals and defined populations" (114). Structurally, the overall clinical culture needs to be welcoming, with the clinical team being advocates to address barriers affecting high quality, care across each and all populations (115).

At the structural level, there may be a cultural divide that needs to be recognized. According to the AUA census, $91 \%$ of urologists in the US are male, $84 \%$ white, $96 \%$ non-Hispanic, $73 \%$ aged over 45 years, and they disproportionately work in the Southeast (116). From discussions with residency directors, the specialty may disproportionately attract students who are most interested in and comfortable with being good technologists, preferring the excitement of surgery and technological advances over talking with patients and follow-up care. Unfortunately, an older, white, heterosexual, male dominant, technology-focused profession may be structurally illequipped to advance the treatment needs of minorities. This suggests that urology-as-a-culture may need to evolve as the proportion of SGM patients increases. The culture has made significant advances in cultural sensitivity to racial and ethnic groups. Now, it needs to broaden those successes to include the groups discussed in this article.

Training is a key component to this (117). To advance cultural humility training in the care of SGM prostate cancer patients, it is insufficient to simply provide a basic, introductory "SGM 101" seminar or case review of an SGM patient. Residency, fellowship, and continuing education training needs to be evidence-informed, as summarized in this paper. Revision of medical school curricula to include the health care needs of SGM is essential to producing patient centered physicians. A recent survey demonstrated only 31 percent of medical students currently receive such training (118).

As defined in the introduction, the goal of evidenceinformed, culturally relevant care is a health care system and workforce that can deliver the highest quality of care to every patient by tailoring treatment to that patient's or population's needs. While the differences in provision of minority versus majority care can seem overwhelming at first, it also makes clinical practice more varied and exciting. Ultimately, if patients are clinician's best teachers, then diverse patients have the greatest potential to improve clinical practice both for individual providers and ultimately for the specialty as a whole.

\section{Acknowledgments}

We would like to acknowledge our community partner, Malecare, for providing online support services for SGM with prostate cancer and for help with recruitment in Restore-1 and Restore-2.

Funding: This study was supported by funding from the National Cancer Institute (NCI) [Grant awards: 1R21CA182041 and 1R01CA218657]. The content is solely the responsibility of the authors and does not necessarily represent the official views of the National Institutes of Health.

\section{Footnote}

Provenance and Peer Review: This article was commissioned by the editorial office, Translational Andrology and Urology for the series "Current and Future Topics on Prostate Cancer". The article has undergone external peer review.

Peer Review File: Available at http://dx.doi.org/10.21037/ tau-20-1052

Conflicts of Interest: All authors have completed the ICMJE uniform disclosure form (available at http://dx.doi. org/10.21037/tau-20-1052). The series "Current and Future Topics on Prostate Cancer" was commissioned by the editorial office without any funding or sponsorship. BRK serves as an unpaid editorial board member of Translational 
Andrology and Urology and served as the unpaid Guest Editor of the series. The authors have no other conflicts of interest to declare.

Ethical Statement: The authors are accountable for all aspects of the work in ensuring that questions related to the accuracy or integrity of any part of the work are appropriately investigated and resolved.

Open Access Statement: This is an Open Access article distributed in accordance with the Creative Commons Attribution-NonCommercial-NoDerivs 4.0 International License (CC BY-NC-ND 4.0), which permits the noncommercial replication and distribution of the article with the strict proviso that no changes or edits are made and the original work is properly cited (including links to both the formal publication through the relevant DOI and the license). See: https://creativecommons.org/licenses/by-nc-nd/4.0/.

\section{References}

1. Quinn GP, Sutton SK, Winfield B, et al. Lesbian, Gay, Bisexual, Transgender, Queer/Questioning (LGBTQ) Perceptions and Health Care Experiences. J Gay Lesbian Soc Serv 2015;27:246-61.

2. Lisy K, Peters MDJ, Schofield P, et al. Experiences and unmet needs of lesbian, gay, and bisexual people with cancer care: A systematic review and meta-synthesis. Psychooncology 2018;27:1480-9.

3. Rosser BRS, Hunt SL, Capistrant BD, et al. Understanding Prostate Cancer in Gay, Bisexual, and Other Men Who Have Sex with Men and Transgender Women: A Review of the Literature. Curr Sex Health Rep 2019;11:430-41.

4. McInnis MK, Pukall CF. Sex After Prostate Cancer in Gay and Bisexual Men: A Review of the Literature. Sex Med Rev 2020;8:466-72.

5. JamesWorsley A, Alexis O. Synthesizing qualitative studies exploring gay and bisexual men's experiences of prostate cancer. Thousand Oaks (CA): SAGE, 2020.

6. Griggs J, Maingi S, Blinder V, et al. American Society of Clinical Oncology Position Statement: Strategies for Reducing Cancer Health Disparities Among Sexual and Gender Minority Populations. J Clin Oncol 2017;35:2203-8.

7. Hart TL, Coon DW, Kowalkowski MA, et al. Changes in sexual roles and quality of life for gay men after prostate cancer: challenges for sexual health providers. J Sex Med 2014;11:2308-17.
8. Ussher JM, Perz J, Kellett A, et al. Health-Related Quality of Life, Psychological Distress, and Sexual Changes Following Prostate Cancer: A Comparison of Gay and Bisexual Men with Heterosexual Men. J Sex Med 2016;13:425-34.

9. Rosser BRS, Kohli N, Polter EJ, et al. The Sexual Functioning of Gay and Bisexual Men Following Prostate Cancer Treatment: Results from the Restore Study. Arch Sex Behav 2020;49:1589-600.

10. Motofei IG, Rowland DL, Popa F, et al. Preliminary study with bicalutamide in heterosexual and homosexual patients with prostate cancer: a possible implication of androgens in male homosexual arousal. BJU Int 2011;108:110-5.

11. Lee TK, Breau RH, Eapen L. Pilot study on quality of life and sexual function in men-who-have-sex-with-men treated for prostate cancer. J Sex Med 2013;10:2094-100.

12. Wassersug RJ, Lyons A, Duncan D, et al. Diagnostic and outcome differences between heterosexual and nonheterosexual men treated for prostate cancer. Urology 2013;82:565-71.

13. Allensworth-Davies D, Talcott JA, Heeren T, et al. The Health Effects of Masculine Self-Esteem Following Treatment for Localized Prostate Cancer Among Gay Men. LGBT Health 2016;3:49-56.

14. Amarasekera C, Wong V, Jackson K, et al. A Pilot Study Assessing Aspects of Sexual Function Predicted to Be Important After Treatment for Prostate Cancer in Gay Men: An Underserved Domain Highlighted. LGBT Health 2020;7:271-6.

15. Dorff TB, Shazer RL, Nepomuceno EM, et al. Successful treatment of metastatic androgen-independent prostate carcinoma in a transsexual patient. Clin Genitourin Cancer 2007;5:344-6.

16. Miksad RA, Bubley G, Church P, et al. Prostate cancer in a transgender woman 41 years after initiation of feminization. JAMA 2006;296:2316-7.

17. Thurston AV. Carcinoma of the prostate in a transsexual. Br J Urol 1994;73:217.

18. Turo R, Jallad S, Prescott S, et al. Metastatic prostate cancer in transsexual diagnosed after three decades of estrogen therapy. Can Urol Assoc J 2013;7:E544-6.

19. van Haarst EP, Newling DW, Gooren LJ, et al. Metastatic prostatic carcinoma in a male-to-female transsexual. Br J Urol 1998;81:776.

20. Hart S, Coon D, Kowalkowski M, et al. Gay men with prostate cancer report significantly worse HRQOL than heterosexual men. J Urol 2011;185:e68-e9.

21. Rose D, Ussher JM, Perz J. Let's talk about gay sex: gay 
and bisexual men's sexual communication with healthcare professionals after prostate cancer. Eur J Cancer Care (Engl) 2017. doi: 10.1111/ecc.12469.

22. Ussher JM, Perz J, Rose D, et al. Threat of Sexual Disqualification: The Consequences of Erectile Dysfunction and Other Sexual Changes for Gay and Bisexual Men With Prostate Cancer. Arch Sex Behav 2017;46:2043-57.

23. Rosser BRS, Capistrant B, Torres MB, et al. The effects of radical prostatectomy on gay and bisexual men's sexual functioning and behavior: qualitative results from the restore study. Sex Relation Ther 2016;31:432-45.

24. Rosser BR, Capistrant B, Torres B, et al. The Effects of Radical Prostatectomy on Gay and Bisexual Men's Mental Health, Sexual Identity and Relationships: Qualitative Results from the Restore Study. Sex Relation Ther 2016;31:446-61.

25. West WG, Rosser BRS, Capistrant BD, et al. The effects of radiation therapy for prostate cancer on gay and bisexual men's mental health, sexual functioning and behavior, sexual identity and relationships: Qualitative results from the Restore study. In: Ussher J, Perz J, Rosser BRS, editors. Gay and Bisexual Men Living with Prostate Cancer: From Diagnosis to Recovery. New York, NY: Harrington Park Press, 2018:216-29.

26. Capistrant BD, Torres B, Merengwa E, et al. Caregiving and social support for gay and bisexual men with prostate cancer. Psychooncology 2016;25:1329-36.

27. Dewitt J, Capistrant B, Kohli N, et al. Addressing Participant Validity in a Small Internet Health Survey (The Restore Study): Protocol and Recommendations for Survey Response Validation. JMIR Res Protoc 2018;7:e96.

28. Polter EJ, Wheldon CW, Rosser BRS, et al. Health-related quality of life by human immunodeficiency virus status in a cross-sectional survey of gay and bisexual prostate cancer survivors. Psychooncology 2019;28:2351-7.

29. Rosser BRS, Konety BR, Capistrant BD, et al. The reliability of Gleason scores in studies of gay, bisexual and other men who have sex with men prostate cancer survivors. Urol Pract 2020. In press.

30. Rosser BRS, Konety BR, Kohli N, et al. What gay and bisexual prostate cancer patients want in a sexual rehabilitation program for prostate cancer: Results of the Restore needs assessment. Urol Pract 2018;5:192-7.

31. Rosser BRS, Konety BR, Mitteldorf D, et al. What gay and bisexual men treated for prostate cancer are offered and attempt as sexual rehabilitation for prostate cancer: Quantitative results from the Restore study with implications for clinicians. Urol Pract 2018;5:187-91.

32. Talley KM, Polter E, Capistrant BD, et al. Lower urinary tract and bowel symptoms predict quality of life in gay and bisexual prostate cancer survivors. Innov Aging 2019;3:S490-1.

33. Rosser BRS, Capistrant BD, Kohli N, et al. Restore: Improving sexual outcomes of gay and bisexual prostate cancer survivors (Grant \# 1R01CA218657-01). Available online: https://clinicaltrials.gov/ct2/show/NCT03343093

34. Rosser BRS, Polter EJ, Chandiramani N, et al. The acceptability and feasibility of collecting sexual orientation and gender identity data in urology and oncology clinics. LGBT Health. Forthcoming 2021.

35. Sexual and Gender Minority Research Office (SGMRO), National Institutes of Health (NIH). Sexual and Gender Minority Populations in NIH-supported Research (NOTOD-19-139). 2019.</unknown>

36. Notice of Special Interest in Research on the Health of Sexual and Gender Minority (SGM) Populations [database on the Internet]. DHHS. 2019. Available online: https://grants.nih.gov/grants/guide/notice-files/NOTMD-19-001.html. Accessed: March 26, 2019.

37. Alexander R, Parker K, Schwetz T. Sexual and Gender Minority Health Research at the National Institutes of Health. LGBT Health 2016;3:7-10.

38. The Williams Institute, UCLA School of Law. LGBT Demographic Data Interactive. UCLA, Los Angeles, CA. 2020. Available online: https://williamsinstitute.law. ucla.edu/visualization/lgbt-stats/?topic=LGBT\#density. Accessed January 242020.

39. Meerwijk EL, Sevelius JM. Transgender Population Size in the United States: a Meta-Regression of Population-Based Probability Samples. Am J Public Health 2017;107:e1-8.

40. Newport F. In US, estimate of LGBT population rises to 4.5\%. 2018.</unknown>

41. National Cancer Institute (NCI), Surveillance E, and End Results Program. Cancer stat facts: Prostae cancer 2020. Available online: https:// seer.cancer.gov/statfacts/html/ prost.html

42. Meyer IH. Prejudice and discrimination as social stressors. In: Meyer IH, Northridge ME, editors. The Health of Sexual Minorities. Washington, DC: APA, 2007.

43. Meyer IH, Northridge ME. The health of sexual minorities: Public health perspectives on lesbian, gay, bisexual and transgender populations. New York, NY: Springer, 2007.

44. Riggle ED, Whitman JS, Olson A, et al. The positive aspects of being a lesbian or gay man. Prof Psychol Res Pr 
2008;39:210

45. Croghan CF, Moone RP, Olson AM. Working With LGBT Baby Boomers and Older Adults: Factors That Signal a Welcoming Service Environment. J Gerontol Soc Work 2015;58:637-51.

46. Fredriksen-Goldsen KI, Muraco A. Aging and Sexual Orientation: A 25-Year Review of the Literature. Res Aging 2010;32:372-413.

47. Wallace SP, Cochran SD, Durazo EM, et al. The health of aging lesbian, gay and bisexual adults in California. Policy Brief UCLA Cent Health Policy Res 2011;(PB2011-2):1-8.

48. Berger RM. Gay and gray: The older homosexual man. New York, NY: Harrington Park Press/Haworth Press, 1996.

49. Doyle BB. Satisfying mature gay sexuality. 2020. Available online: https://www.asaging.org/blog/satisfying-maturegay-sexuality. Accessed September 182020.

50. Cahill S, South K. Policy issues affecting lesbian, gay, bisexual, and transgender people in retirement. Generations 2002;26:49-54.

51. Kurdek LA. Differences between heterosexual-nonparent couples and gay, lesbian and heterosexual-parent cohabitating couples. J Marriage Fam 2001;60:553-68.

52. Buxton AP. Writing our own script: How bisexual men and their heterosexual wives maintain their marriages after disclosure. J Bisex 2000;1:155-89.

53. Rosenberger JG, Reece M, Schick V, et al. Sexual behaviors and situational characteristics of most recent malepartnered sexual event among gay and bisexually identified men in the United States. J Sex Med 2011;8:3040-50.

54. Moskowitz DA, Rieger G, Roloff ME. Tops, bottoms and versatiles. Sex Relation Ther 2008;23:191-202.

55. Kutner BA, Simoni JM, King KM, et al. Does Stigma Toward Anal Sexuality Impede HIV Prevention Among Men Who Have Sex With Men in the United States? A Structural Equation Modeling Assessment. J Sex Med 2020;17:477-90.

56. Diaz RM. Latino Gay Men and HIV. New York: Routledge, 1998.

57. Mitteldorf D. Psychotherapy with gay prostate cancer patients. J Gay Lesbian Ment Health 2005;9:56-67.

58. Noor SW, Rosser BR. Enema use among men who have sex with men: a behavioral epidemiologic study with implications for HIV/STI prevention. Arch Sex Behav 2014;43:755-69.

59. Perlman G, Drescher J. Introduction: What gay men (and those near and dear to them) need to know about prostate cancer.J Gay Lesbian Ment Health 2005;9:1-7.

60. Amarasekera C, Cohen J, Wong V, et al. Urologists' knowledge, attitudes and practice behaviors regarding sexual minority patients. Proceedings of the AUA Annual Meeting; Chicago, IL, 2019.

61. Kent EE, Wheldon CW, Smith AW, et al. Care delivery, patient experiences, and health outcomes among sexual and gender minority patients with cancer and survivors: A scoping review. Cancer 2019;125:4371-9.

62. Boehmer U, Miao X, Ozonoff A. Cancer survivorship and sexual orientation. Cancer 2011;117:3796-804.

63. Agenor M. Chapter 8. What are the numbers? The epidemiology of cancer by sexual orientation and gender identity. In: Boehmer U, Elk R, editors. Cancer and the LGBT community: Unique Perspectives from Risk to Survivorship. New York, NY: Springer, 2015:117-40.

64. Boehmer U, Miao X, Maxwell NI, et al. Sexual minority population density and incidence of lung, colorectal and female breast cancer in California. BMJ Open 2014;4:e004461.

65. Baker KC, Durso LE, Cray A. Moving the needle: The impact of the Affordable Care Act on LGBT communities. Center for American Progress; 2014.

66. Blank TO, Descartes L, Asencio M. Cancer screening in gay and bisexual men and transgender people. In: Boehmer U, Elk R. Cancer and the LGBT Community. New York: Springer, 2015:99-114.

67. Capistrant BD, Lesher L, Kohli N, et al. Social Support and Health-Related Quality of Life Among Gay and Bisexual Men With Prostate Cancer. Oncol Nurs Forum 2018;45:439-55.

68. Kamen C, Mustian KM, Dozier A, et al. Disparities in psychological distress impacting lesbian, gay, bisexual and transgender cancer survivors. Psychooncology 2015;24:1384-91.

69. Crangle CJ, Latini DM, Hart TL. The effects of attachment and outness on illness adjustment among gay men with prostate cancer. Psychooncology 2017;26:500-7.

70. White W, Brenman S, Paradis E, et al. Lesbian, Gay, Bisexual, and Transgender Patient Care: Medical Students' Preparedness and Comfort. Teach Learn Med 2015;27:254-63.

71. Margolies L, Brown CG. Current State of Knowledge About Cancer in Lesbians, Gay, Bisexual, and Transgender (LGBT) People. Semin Oncol Nurs 2018;34:3-11.

72. Bauer GR, Scheim AI, Pyne J, et al. Intervenable factors associated with suicide risk in transgender persons: a respondent driven sampling study in Ontario, Canada. BMC Public Health 2015;15:525.

73. Bauer GR, Travers R, Scanlon K, et al. High heterogeneity 
of HIV-related sexual risk among transgender people in Ontario, Canada: a province-wide respondent-driven sampling survey. BMC Public Health 2012;12:292.

74. Scheim AI, Bauer GR, Shokoohi M. Drug use among transgender people in Ontario, Canada: Disparities and associations with social exclusion. Addict Behav 2017;72:151-8.

75. Baral SD, Poteat T, Strömdahl S, et al. Worldwide burden of HIV in transgender women: a systematic review and meta-analysis. Lancet Infect Dis 2013;13:214-22.

76. Reisner SL, Poteat T, Keatley J, et al. Global health burden and needs of transgender populations: a review. Lancet 2016;388:412-36.

77. Bradford J, Reisner SL, Honnold JA, et al. Experiences of transgender-related discrimination and implications for health: results from the Virginia Transgender Health Initiative Study. Am J Public Health 2013;103:1820-9.

78. Bauer GR, Hammond R, Travers R, et al. "I don't think this is theoretical; this is our lives": how erasure impacts health care for transgender people. J Assoc Nurses AIDS Care 2009;20:348-61.

79. Bauer GR, Scheim AI, Deutsch MB, et al. Reported emergency department avoidance, use, and experiences of transgender persons in Ontario, Canada: results from a respondent-driven sampling survey. Ann Emerg Med 2014;63:713-20.e1.

80. Giblon R, Bauer GR. Health care availability, quality, and unmet need: a comparison of transgender and cisgender residents of Ontario, Canada. BMC Health Serv Res 2017;17:283.

81. Lombardi E. Enhancing transgender health care. Am J Public Health 2001;91:869-72.

82. Lombardi EL, Wilchins RA, Priesing D, et al. Gender violence: transgender experiences with violence and discrimination. J Homosex 2001;42:89-101.

83. Grant J, Mottet L, Tanis J, et al. National transgender discrimination survey report on health and health care: Findings of a study by the National Center for Transgender Equality and the National Gay and Lesbian Task Force. National Center for Transgender Equality 2010. Available online: http://transexualia.org/wp-content/ uploadsw/2015/03/Sanidad ntdsreportonhealth.pdf

84. James S, Herman J, Rankin S, et al. The report of the 2015 US transgender survey. 2016. Available online: https:// www/ transequality.org/sites/default/docs/files/docs/ USTS-Full-Report-FINAL.PDF

85. Lombardi E. Varieties of transgender/transsexual lives and their relationship with transphobia. J Homosex
2009;56:977-92.

86. Lombardi E. Transgender health: A review and guidance for future research-Proceedings from the Summer Institute at the Center for Research on Health and Sexual Orientation, University of Pittsburgh. Int J Transgend 2010;12:211-29.

87. Levitt N. Clinical nursing care for transgender patients with cancer. Clin J Oncol Nurs 2015;19:362-6.

88. Meriggiola MC, Berra M. Long-term cross-sex hormone treatment is safe in transsexual subjects. Asian J Androl 2012;14:813-4.

89. Asscheman H, Giltay EJ, Megens JA, et al. A long-term follow-up study of mortality in transsexuals receiving treatment with cross-sex hormones. Eur J Endocrinol 2011;164:635-42.

90. Deebel NA, Morin JP, Autorino R, et al. Prostate Cancer in Transgender Women: Incidence, Etiopathogenesis, and Management Challenges. Urology 2017;110:166-71.

91. Peitzmeier SM, Reisner SL, Harigopal P, et al. Femaleto-male patients have high prevalence of unsatisfactory Paps compared to non-transgender females: implications for cervical cancer screening. J Gen Intern Med 2014;29:778-84.

92. Tabaac AR, Sutter ME, Wall CSJ, et al. Gender Identity Disparities in Cancer Screening Behaviors. Am J Prev Med 2018;54:385-93.

93. Unger CA. Care of the transgender patient: a survey of gynecologists' current knowledge and practice. J Womens Health (Larchmt) 2015;24:114-8.

94. Meyer IH. Minority stress and mental health in gay men. J Health Soc Behav 1995;36:38-56.

95. Higgins G. A gay man and his partner face his prostate cancer together. J Gay Lesbian Ment Health 2005;9:147-53.

96. West W, Torres B, Mitteldorf D, et al. The challenge of coming out to providers by gay and bisexual men with prostate cancer: Qualitative results from the Restore study. Forthcoming 2021.

97. Trofimenko V, Myers JB, Brant WO. Post-Prostatectomy Incontinence: How Common and Bothersome Is It Really? Sex Med Rev 2017;5:536-43.

98. Donovan JL, Hamdy FC, Lane JA, et al. Patient-Reported Outcomes after Monitoring, Surgery, or Radiotherapy for Prostate Cancer. N Engl J Med 2016;375:1425-37.

99. Daugherty M, Chelluri R, Bratslavsky G, et al. Are we underestimating the rates of incontinence after prostate cancer treatment? Results from NHANES. Int Urol Nephrol 2017;49:1715-21. 
100.Avery K, Donovan J, Peters TJ, et al. ICIQ: a brief and robust measure for evaluating the symptoms and impact of urinary incontinence. Neurourol Urodyn 2004;23:322-30.

101. Rosser BRS, Polter E. Prostate cancer in sexual minority men: An analysis of older versus younger gay, bisexual and other men who have sex with men. Proceedings of the Alliance Annual Meeting; 2019 Nov 8; Chicago, IL.

102. Lavery JF, Clarke VA. Prostate cancer: Patients' and spouses' coping and marital adjustment. Health Psychol Behav Med 1999;4:289-302.

103. Gray RE, Fitch M, Phillips C, et al. Managing the Impact of Illness: The Experiences of Men with Prostate Cancer and their Spouses. J Health Psychol 2000;5:531-48.

104. Capistrant BD, Garcia RL, Kohli N, et al. Is mental and physical health associated with relationship satisfaction in gay and bisexual prostate cancer patients and their partners? A pilot dyadic study. Forthcoming, 2021. (In press).

105. Mayer KH, Bradford JB, Makadon HJ, et al. Sexual and gender minority health: what we know and what needs to be done. Am J Public Health 2008;98:989-95.

106. Stein GL, Beckerman NL, Sherman PA. Lesbian and gay elders and long-term care: identifying the unique psychosocial perspectives and challenges. J Gerontol Soc Work 2010;53:421-35.

107.Henning-Smith C, Gonzales G, Shippee TP. Differences by Sexual Orientation in Expectations About Future LongTerm Care Needs Among Adults 40 to 65 Years Old. Am J Public Health 2015;105:2359-65.

108. King S. Addressing the health needs of older gays and lesbians. Springville (UT): Liberty Press, 2009;15:25-8. 109. Erdley SD, Anklam DD, Reardon CC. Breaking barriers

Cite this article as: Rosser BRS, Rider GN, Kapoor A, Talley KMC, Haggart R, Kohli N, Konety BR, Mitteldorf D, Polter EJ, Ross MW, West W, Wheldon C, Wright M. Every urologist and oncologist should know about treating sexual and gender minority prostate cancer patients: translating research findings into clinical practice. Transl Androl Urol 2021;10(7):3208-3225. doi: $10.21037 /$ tau-20-1052 and building bridges: understanding the pervasive needs of older LGBT adults and the value of social work in health care. J Gerontol Soc Work 2014;57:362-85.

110.Span P. A retirement community turned away these married women. New York Times. 2018 Aug 20, 2018. Available online: https://www.nytimes.com/2018/08/17/ health/ lgbt-discrimination-retirement.html

111. McFarland PL, Sanders S. A pilot study about the needs of older gays and lesbians: What social workers need to know. J Gerontol Soc Work 2003;40:67-80.

112. Knauer NJ. LGBT elder law: Toward equity in aging. Harv J Law 2009;32:1.

113.Dowsett GW. "Losing my chestnut": One gay man's wrangle with prostate cancer. Reprod Health Matters 2008;16:145-50.

114. Tervalon M, Murray-García J. Cultural humility versus cultural competence: a critical distinction in defining physician training outcomes in multicultural education. J Health Care Poor Underserved 1998;9:117-25.

115. Kleinman A, Benson P. Anthropology in the clinic: the problem of cultural competency and how to fix it. PLoS Med 2006;3:e294.

116.American Urological Association. The state of the urology workforce and practice in the United States 2018. Linthicum, MD, 2019.

117.Alpert A, Kamen C, Schabath MB, et al. What Exactly Are We Measuring? Evaluating Sexual and Gender Minority Cultural Humility Training for Oncology Care Clinicians. J Clin Oncol 2020;38:2605-9.

118.Jenkins MR, Herrmann A, Tashjian A, et al. Sex and gender in medical education: a national student survey. Biol Sex Differ 2016;7:45. 